\section{$\underset{\substack{\text { hommes } \\ \text { \& migrations }}}{ }$}

\section{Hommes \& migrations}

Revue française de référence sur les dynamiques

migratoires

$1314 \mid 2016$

Migrations chinoises et générations

\title{
Femme et mère, entre le besoin et le désir
}

Étude de cas clinique d'une femme chinoise se prostituant à Paris

\section{Ting Chen}

\section{(2) OpenEdition \\ 1 Journals}

\section{Édition électronique}

URL : http://journals.openedition.org/hommesmigrations/3644

DOI : 10.4000/hommesmigrations.3644

ISSN : 2262-3353

Éditeur

Musée national de l'histoire de l'immigration

\section{Édition imprimée}

Date de publication : 1 avril 2016

Pagination : 93-100

ISBN : 978-2-919040-35-3

ISSN : 1142-852X

\section{Référence électronique}

Ting Chen, « Femme et mère, entre le besoin et le désir », Hommes \& migrations [En ligne], 1314 | 2016, mis en ligne le 01 avril 2019, consulté le 19 avril 2019. URL : http://journals.openedition.org/

hommesmigrations/3644; DOI : 10.4000/hommesmigrations.3644 


\title{
DES PERSONNES ÂGÉES D'ORIGINE CHINOISE À BELLEVILLE \\ PARCOURS MIGRATOIRES, DYNAMIQUES FAMILIALES ET PARTICIPATION À LA VIE DU QUARTIER
}

\author{
par SIMENG WANG, docteure en sociologie diplômée à l'ENS-EHESS (CMH) \\ et actuellement post-doctorante contractuelle IFRIS affectée au CEPED, et BORIS SCHWARTZ, \\ médiateur à l'association Chinois de France - Français de Chine (CFFC).
}

\begin{abstract}
Les personnes âgées d'origine chinoise vivant dans le quartier parisien de Belleville sont peu visibles dans l'espace public. Deux enquêtes qualitatives tentent d'éclairer la diversité de leurs conditions de vie et de leurs trajectoires migratoires. Leur invisibilité peut s'expliquer par l'existence d'entraves linguistiques et par les différentes formes de discriminations qu'elles ressentent. Par rapport aux hommes, les femmes chinoises âgées constituent davantage le pivot de l'interface avec la société d'accueil. Il demeure toutefois difficile de parler d'un isolement de ces personnes âgées, notamment en tenant compte de la sociabilité au sein des réseaux ethniques.
\end{abstract}

Les personnes âgées d'origine chinoise qui fréquentent le quartier de Belleville demeurent méconnues. Afin de dévoiler la diversité de leurs conditions de vie, deux corpus de matériaux empiriques collectés dans le cadre des activités de l'association Chinois de France - Français de Chine (CFFC) ont été mobilisés. Le premier consiste en une enquête conjointement conçue par trois types d'acteurs : des membres de l'association, des responsables politiques de la Ville de Paris, notamment du quartier de Belleville ${ }^{1}$, et une sociologue travaillant sur l'immigration chinoise en France.
L'enquête se propose de cerner les profils de ces personnes âgées non francophones et peu visibles dans l'espace public. Le second corpus vient d'une étude menée au sein de l'association CFFC auprès de seniors qui fréquentent la structure au travers des activités qui leur sont ouvertes (cours de français, réunions médicales, permanences sociales, sorties culturelles). Bien que leurs méthodes d'enquête soient différentes, ces deux dispositifs d'observation s'avèrent complémentaires pour appréhender une population peu visible dans l'espace public. 


\section{Genèse et objectif de l'enquête collective "Seniors chinois"}

Inspirée par l'association BATIK et son projet nommé "Patriarches" qui vise à recueillir les témoignages de migrants âgés vivant dans le XIII arrondissement, principalement originaires du Maghreb, d'Afrique subsaharienne et d'Asie du Sud-Est, l'association CFFC basée dans le quartier de Belleville a souhaité expérimenter la même initiative auprès des personnes âgées originaires de la Chine continentale. Subventionnée par la Ville de Paris, cette association met en avant dans son questionnement la dimension "diagnostic de territoire". Il s'agit d'identifier et de connaitre l'ensemble des ressources du quartier de Belleville mobilisées autour des seniors chinois, et de mieux

Il s'agit d'identifier

et de connaître l'ensemble

des ressources du quartier

de Belleville mobilisées

autour des seniors chinois,

et de mieux comprendre

le quotidien de ces personnes, les difficultés éventuellement rencontrées et leurs attentes. comprendre le quotidien de ces personnes, les difficultés éventuellement rencontrées et leurs attentes. L'association sollicite la sociologue Simeng Wang qui a consacré ces dernières années à la question de la prise en charge sanitaire en particulier sur le plan de la santé mentale - de la population chinoise résidant à Paris. Elle n'a jusque-là rencontré que quelques cas de patients du troisième âge (parmi 180 cas rencontrés ${ }^{2}$ ), mais s'intéresse à la prise en charge sanitaire et sociale de cette population. En outre, étudier les conditions de vie des personnes âgées permet d'apporter un autre regard sur les relations intergénérationnelles, la structure familiale et les dynamiques familiales au sein des foyers chinois d'outre-mer. Les centres d'intérêts de l'association $C F F C$ et de la sociologue rejoignent les attentes des responsables politiques de la Ville de Paris. Lors d'une réunion ${ }^{3}$, ces derniers font part de leur intérêt pour ces personnes âgées à la fois "non francophones" et "peu visibles dans l'espace public", avec le souci de leur prise en charge future à la fois sanitaire et sociale.

En juillet 2014, deux membres de l'association CFFC, Tamara Lui (Française d'origine cantonaise, immigrée en France au début des années 1990) et l'ancien coordinateur, Yves Traynard (Français, ancien cadre dans le secteur privé, amateur de la Chine, ayant eu l'expérience de l'enseignement du français dans une université chinoise), et Simeng Wang sont chargés du pilotage de l'enquête $^{4}$. Létude se focalise sur les Chinois originaires de la République populaire de Chine et, dans un premier temps, sur ceux visibles dans l'espace public. Il s'agit des personnes âgées qui suivent des cours de français dans cette association, qui y font leurs courses ou qui participent à des activités de loisirs en plein air telles que la gymnastique, la danse (mais pas celles qui jouent au majong à leur domicile, par exemple). Pour l'instant, l'entrée par les institutions - cliniques, hôpitaux, foyers, etc. n'est pas encore explorée comme une piste de rencontre des personnes âgées. Ce choix de la modalité de recrutement des enquêtés exclut des personnes âgées dites "dépendantes".

Nous établissons dans un premier temps un questionnaire en français ${ }^{5}$ que nous traduisons en mandarin. Seule la version chinoise du questionnaire est utilisée durant l'enquête. Quatre bénévoles de l'association - trois femmes et un homme, tous Chinois nés en Chine et arrivés en France après l'an 2000 - participent à la collecte des matériaux. En

2. Simeng Wang, "Expériences migratoires au prisme des usages des soins psychiatriques. Le cas de l'immigration chinoise en région parisienne. Une enquête ethnographique en institution et dans les familles", thèse de doctorat, ENS-EHESS, 2014. 3. Cette réunion a eu lieu en amont de la collaboration de la sociologue avec l'association CFFC sur l'enquête "Seniors chinois". La rencontre s'est déroulée à l'Hôtel de Ville, le 7 mai 2014, entre Jeanne Bonnemay - alors conseillère technique de Myriam El Khomri, qui était quant à elle adjointe à la Maire de Paris chargée de la sécurité, de la prévention, de la politique de la Ville et de l'Intégration -, Stéphane Beaud - sociologue et un des directeurs de thèse de Simeng Wang - et cette dernière. 4. Au cours de l'établissement du questionnaire, la divergence des intérêts des trois types d'acteurs impliqués est exprimée. Les acteurs du monde associatif souhaitent mettre l'accent sur le développement d'activités associatives. Quant à la sociologue, elle insiste plutôt sur les parcours migratoires des personnes âgées et les réseaux qu'elles maintiennent en France et au-delà, afin de capter les reconfigurations de leurs espaces de vie au cours de leur mobilité géographique. 5. Plusieurs questions posées dans ce questionnaire font écho à l'Enquête 2011 Famille et logement (EFL), I'Insee et l'Ined. 
parallèle de la passation de ce questionnaire auprès d'une trentaine de seniors, une vingtaine d'observations participantes dans leurs lieux de sociabilité (tels que les parcs et cafés) et dix-sept entretiens semi-directifs individuels ou collectifs auprès de personnes âgées (onze femmes et six hommes) et de membres de leur famille ont été effectués. Le taux de refus de participation à l'enquête étant très élevé - seule une personne âgée sur dix l'accepte -, nous constatons une forte méfiance de la population d'étude envers cette recherche. Nous devons ainsi recourir à la méthode "boule de neige" et établir des relations de confiance avec ces personnes âgées grâce aux longs moments passés en observation participante. Nous tenons à préciser que cette enquête inaugurée en 2014 est toujours en cours et, qu'au stade actuel, nous ne prétendons pas offrir aux lecteurs des résultats représentatifs concernant toute la population âgée d'origine chinoise du quartier de Belleville. Il s'agit en revanche de partager nos premières analyses de la pré-enquête, de nature plutôt qualitative.

\section{Les matériaux collectés au sein de l'association CFFC}

Cette seconde étude repose sur une cinquantaine d'observations et de témoignages recueillis auprès de quarante personnes âgées fréquentant des permanences socio-juridiques, ouvertes deux fois par semaine sans rendez-vous à tout type de migrants au sein de l'association CFFC. À cela s'ajoutent vingt questionnaires distribués auprès de seize femmes et quatre hommes. Dans la mesure où ces seniors viennent demander un service (dans le cadre de la permanence d'écrivain public, par exemple) ou que nous les rencontrons régulièrement dans le cadre des ateliers de langue, une réelle proximité s'établit entre eux et les salariés et bénévoles de l'association. Cela a contribué à un faible taux de refus de participation à l'enquête ${ }^{6}$, contrairement au dispositif précédent.
Au sein de l'association, nous constatons que la fréquentation des activités par les personnes âgées est de plus en plus importante, ce qui traduit leur demande d'accompagnement social. Il s'agit d'aider et de conseiller ces personnes âgées dans leurs démarches vers le système de sécurité sociale en particulier ou auprès de l'administration en général. Un autre constat porte sur la plus forte participation des femmes âgées à l'association. Parmi les vingt répondants du questionnaire, seuls quatre sont des hommes, dont deux participent à l'atelier de langue française, accompagnés de leur femme. Cela révèle la plus grande difficulté d'intéresser le public senior masculin.

Lâge moyen de ces personnes
Du fait de ce système de répartition des rôles sociaux, les femmes chinoises âgées se trouvent plus souvent que les hommes en première ligne de l'interface avec la société d'accueil, malgré la barrière de la langue. est de 65 ans. Les deux tiers sont originaires de la province du Zhejiang, notamment de la municipalité de Wenzhou. Quant aux autres, ils viennent de la province du Shandong située au nord-est de la Chine, et des régions du Sud-Ouest, que sont le Yunnan et le Sichuan. Certaines personnes âgées, installées de longue date, sont soutenues par leur famille et en demande d'activités socioculturelles. Alors que d'autres vivent dans des situations plus précaires : isolées, elles sont en quête d'informations sur leurs droits sociaux.

\section{Profils et rôles genrés des seniors chinois}

L'année de migration et la région de provenance s'avèrent pertinentes pour dégager des profils types de ces seniors chinois et mettre en lumière leur parcours migratoires. La plupart des migrants originaires de la région de Wenzhou sont arrivés en France, à quelques exceptions près, dans les années 1980, au début de la politique de réformes et d'ouverture de la Chine. Paysans avant leur 
émigration, ils ont travaillé à leur arrivée en France comme petites mains des ateliers de confection de maroquinerie et de prêt-à-porter en Île-de-France. Par la suite, ils ont été, pour la plupart, rejoints par leurs enfants, auprès desquels ils vivent aujourd'hui. Même si leur niveau de vie demeure modeste, les soutiens familiaux les protègent de l'isolement. En revanche, les personnes provenant d'autres régions de Chine - du littoral nord comme le Shandong ou de provinces plus reculées telles que le Sichuan ou le Yunnan - sont souvent arrivées en France après 2010. Leur origine sociale est plus variée, allant de professeur d'université, fonctionnaire local, à ouvrier du textile. Contrairement au premier groupe, ces personnes, retraitées au moment de leur départ, ont souvent été précédées de leurs enfants qu'elles ont rejoints.

L'ancienneté de la migration entraîne un certain nombre de conséquences, notamment du point de vue administratif. Les premiers ont acquis leur titre de séjour et peuvent bénéficier d'une carte de résident de longue durée. Les seconds, qui n'obtiennent que des titres renouvelables d'une année, doivent se mobiliser à échéance régulière pour réunir les justificatifs et renouveler les mêmes démarches administratives auprès de la Préfecture. L'ancienneté de la migration se traduit par une stabilité qui se répercute notamment au niveau de l'assurance médicale. Il n'est pas rare de voir que les personnes arrivées récemment en France passent d'un régime d'assurance à un autre selon la régularisation de leur situation. Ils alternent entre CMU-C et AME, et connaissent des périodes sans aucune couverture médicale.

La caractéristique genrée des enquêtés mérite d'être soulignée. Dans la première enquête, nous avons rencontré quasiment tous les hommes enquêtés dans des lieux de sociabilité tels quau café et au parc, alors que nous sommes plutôt entrés en contact avec les femmes par le biais associatif. Ce sont des femmes du troisième âge qui viennent régulièrement aux cours de français, parfois avec leurs petits-enfants. Comme elles le disent: "Apprendre le français, ça aide au quotidien : faire les courses, aller chercher les petits à l'école, voir le médecin." En plus de l'aspect utile de l'apprentissage du français, certaines enquêtées considèrent les cours comme un lieu de sociabilité : "C'est loccasion de se retrouver avec des connaissances et des amies." Cette modalité genrée de rencontre des personnes âgées chinoises dans l'espace public confirme l'attribution genrée des rôles sociaux : les femmes assurent davantage les tâches domestiques y compris la prise en charge des petitsenfants alors que les hommes ont plus tendance à passer leur temps dans les pratiques de loisirs. Du fait de ce système de répartition des rôles sociaux, les femmes chinoises âgées se trouvent plus souvent que les hommes en première ligne de l'interface avec la société d'accueil, malgré la barrière de la langue. Les hommes chinois du troisième âge, sans activité professionnelle, se sociabilisent pour leur part à l'extérieur du foyer, mais le plus souvent à l'intérieur de leur réseau ethnique, c'est-à-dire du monde chinois. La seconde enquête a été menée dans le cadre des activités de l'association dont les permanences sociales sont fréquentées à $55 \%$ par des personnes âgées de plus de 50 ans, et à hauteur de 21\% par les plus de 60 ans. Parmi elles, les femmes représentent environ les deux tiers, contre un tiers pour les hommes. Il n'est pas rare que la femme vienne au nom de son mari pour des démarches qui le concernent. Les sollicitations, qui touchent à la question de l'accès aux droits sociaux, comprenant la santé, les allocations, la retraite, relèvent en somme des affaires du foyer. C'est donc à la femme que revient le rôle de s'assurer que, sur le plan administratif, tout soit en règle et de s'informer sur ce à quoi le foyer peut prétendre. De même, dans le cadre des activités proposées aux seniors, les femmes sont surreprésentées. Lors des contacts pris avec des hommes, peu d'intérêt a émergé de leur part et l'interaction a été plus brève qu'avec les femmes seniors auprès de qui un véritable dialogue s'est instauré. Elles expriment directement une demande pour des activités qui vien- 
draient compléter leur emploi du temps. Cette posture plus ouverte s'explique sans doute du fait d'un rôle genré qui leur a permis de développer une posture moins distanciée avec le monde "extérieur” de la famille dans la société d'accueil.

\section{Renouvellement de la solidarité familiale intergénérationnelle dans un contexte migratoire}

Un des premiers résultats tirés de notre enquête a souligné la force de la solidarité familiale intergénérationnelle. Ces personnes âgées jouent un rôle important dans la prise en charge de leurs petits-enfants. En moyenne, elles consacrent plus de 4 heures par jour à la garde des petits-enfants.
Le discours de Mme Xu est révélateur : "Je garde son enfant [l'enfant de sa fille], c'est ce qui entretient notre relation. Comme ça, on se voit tous les jours." Si la garde d'enfant participe à l'entretien du lien familial, l'espace géographique y fait également sens. Dans trois quarts des cas, les trois générations habitent sous le même toit ${ }^{7}$. Pour le reste, les personnes âgées habitent souvent dans le même quartier que leurs enfants. La distance entre leurs logements ne dépasse jamais un kilomètre. La fréquence de la réunion familiale reflète également l'intensité du lien familial : les familles qui se réunissent le moins fréquemment (entre les personnes du troisième âge et leurs descendants) se voient une fois toutes les deux semaines. Si le rôle des grands-parents dans la prise en charge des petits-enfants peut s'observer également chez les 
migrants d'autres origines ethniques, une spécificité propre à ces familles chinoises mérite d'être soulignée. La modalité d'immigration en plusieurs temps entre différentes générations fait que les personnes âgées immigrées en France pour raison de "regroupement familial" manifestent un devoir de solidarité familiale envers leurs

La modalité d'immigration en plusieurs temps entre

différentes générations fait que les personnes âgées

immigrées en France pour raison de "regroupement familial" manifestent un devoir de solidarité familiale envers leurs enfants. enfants. Pour M. Pei, il s'agit de répondre à ce qu'il considère comme une dette à l'égard de son fils : "Je le lui dois, c'est lui qui m'a fait venir." Ces personnes âgées ont pu venir en France grâce au statut de leurs enfants - statut juridique de citoyen français et/ou statut socio-économique de propriétaire de biens - et se sentent ainsi "redevables". Pour rendre ce "don" dû à l'immigration ${ }^{8}$, les personnes âgées rencontrées déchargent leurs enfants de divers services : outre la garde des petits-enfants, elles s'occupent du ménage et contribuent à l'économie familiale par leurs ressources : allocations, pension retraite, etc. Une partie de leurs revenus est dépensée pour les courses alimentaires de toute la famille et le reste est souvent remis aux enfants. Dans d'autres circonstances, à défaut de bénéficier d'une assurance retraite ou de revenus de solidarité, un petit pécule est versé par les enfants aux parents pour assurer les dépenses quotidiennes de la famille. À nouveau, nous observons comment les parents assurent un service qui profite à toute la famille, pendant que les enfants sont au travail pour garantir l'économie du foyer. Sans aucun doute, la présence de membres de la famille, qu'il y ait ou non cohabitation, constitue une ressource importante pour les personnes âgées. La famille représente une ressource économique par le niveau de revenu, linguistique par le degré de maîtrise de la langue, et sociale par le degré de connaissance du système administratif et social français. En effet, les personnes rencontrées qui paraissent les plus "précaires", notamment du point de vue économique, sont celles qui ne bénéficient d'aucun soutien familial. Cette précarité s'aggrave au moment de la retraite, où seul le faible nombre d'années travaillées déclarées - le temps de leur régularisation administrative oblige - sera pris en compte pour le calcul de leur pension de retraite. Très souvent, ces pensions, complémentaires comprises, s'élèvent difficilement au-dessus de 200 euros mensuels. C'est alors le maintien dans l'emploi de l'autre conjoint qui permet d'éviter une grande précarité. Cette distinction entre ceux bénéficiant d'un appui familial et les autres semble fondamentale pour comprendre les problèmes sociaux auxquels font face les seniors migrants : précarité économique, difficultés d'accès aux droits sociaux et au logement. À ce faible niveau de ressources, certaines personnes âgées vivent dans des logements insalubres et souffrent de pathologies développées à la suite de conditions de vie précaires.

\section{Participation à la vie du quartier : entraves linguistiques et discriminations ressenties}

Un autre résultat préliminaire qui ressort de cette enquête consiste à interroger les frontières matérielles et symboliques entre l'espace public (gong) et l'espace privé (si) ; entre ce qui est qualifié comme autrui (ta) et ce qui est qualifié comme soi (wo). 
Les personnes que nous avons rencontrées entretiennent peu de relations avec d'autres populations ethniques habitant le quartier, qu'elles soient commerçantes ou voisines. Elles fréquentent dans la plupart des cas les commerces chinois, allant de la boucherie, du supermarché au restaurant et au traiteur. Lorsqu'il s'agit d'un commerce tenu par une connaissance ou interconnaissance de la personne âgée, quelques discussions de la vie courante se développent. Si ce n'est pas le cas, il n'y a pas forcément de discussion entre les deux Chinois. Pourtant, le marché de Belleville qui se tient deux fois par semaine offre à certains seniors chinois l'occasion d'interagir avec les commerçants issus d'ethnies différentes.

Le café tabac de la rue de Belleville, où les hommes chinois seniors apprécient de se retrouver, est aussi un lieu de rencontre potentiel avec d'autres habitants du quartier. Cela ne signifie pas pour autant que les différentes populations, et en particulier les seniors, entretiennent de forts liens entre elles. De la même façon, cette distance s'observe au parc de Belleville qui constitue un espace de sociabilité pour des seniors d'origines différentes, mais où les relations se cantonnent à des échanges de regards.

D’une manière générale, malgré ces différents lieux de contact potentiels entre les seniors chinois et d'autres habitants du quartier, nous notons une forte caractéristique d'"entre-soi" dans la sociabilité des personnes âgées. Même à l'occasion des festivités, tels que les défilés du Nouvel An chinois, toutes les personnes âgées rencontrées n'y participent pas. Cela est encore moins le cas pour ce qui est de la citoyenneté ou du sentiment d'appartenance nationale: aucune de ces personnes dit se sentir concernée par une participation à la vie politique en France. Plus précisément, même celles et ceux qui sont naturalisés n'ont jamais voté. La plupart d'entre eux n'ont pas participé non plus aux manifestations qui ont eu lieu à Belleville en 2010 et 2011.

L'invisibilité de ces personnes âgées dans l'espace public et leur passivité dans la participa- tion aux luttes pour la défense de leurs droits peuvent s'expliquer par l'existence d'entraves linguistiques et par les différentes formes de discriminations quelles ont ressenties, qui freinent conjointement leur démarche de sociabilité avec l'ensemble des membres de la société d'accueil. Aucune de ces personnes âgées ne maîtrise le français. Cela justifie la nature "sinophone" des lieux qu'elles fréquentent : faire des courses dans les supermarchés ou épiceries asiatiques ; sortir jardinage, promenade, danse, gymnastique - avec des compatriotes chinois ; même à la maison ne regarder que les chaînes chinoises à la télévision.

D'une part, nous observons une sociabilité restreinte de ces personnes âgées, limitée à un environnement linguistiquement, ethniquement et socialement homogène. D'autre part, elles expriment un sentiment d'insécurité dans l'interaction avec la population française en général. Ce sentiment est nourri par des expériences de discrimination, de signes de mépris (des regards et des gestes), d'agressions verbales ou physiques (le plus souvent des vols). M. Liu explique ses choix de sorties en ces termes: "Comme mes copains, j'évite d'aller dans les endroits publics. Sauf lorsque c'est vraiment nécessaire, je préfère ne pas sortir de mon quartier [Belleville]. Parce qu'ici, si jamais il marrive quelque chose, des accidents ou des problèmes de santé, je peux toujours trouver des Chinois parmi les passants,
Les personnes que nous avons rencontrées entretiennent peu de relations avec d'autres populations ethniques habitant le quartier, qu'elles soient commerçantes ou voisines. volé dans la rue, je suis même incapable d'appeler la police." Dans le quartier de Belleville, nous constatons une cohabitation avec un minimum d'interaction entre les populations d'origines ethniques différentes. Si ce quartier est considéré comme un microcosme "cosmopolite", il n'existe en réalité pas beaucoup d'échanges entre les Chinois et les autres groupes ethniques, du moins dans le cas des personnes âgées. 


\section{Isolement des personnes âgées : des situations très variées}

Le problème de l'isolement des personnes âgées, qui conjugue une situation de solitude, de dépendance et d'éloignement des proches, est un sujet récurrent dans le débat politique français. Dans le cas des seniors chinois, parler de l'isolement suppose d'appréhender la sociabilité de ce public sous une double perspective.

D'un côté, comme décrit plus haut, l'entre-soi ne conduit pas ces personnes âgées à une situa- tion d'isolement social. Cela fournit une base sur laquelle s'organisent un certain nombre d'activités, tant dans la sphère amicale que familiale : la gymnastique matinale, la promenade du soir, les cérémonies cultuelles. Toutes ces activités occupent, pour la moitié des enquêtés, une place importante dans leur emploi du temps. L'abondance des commerces chinois dans le quartier de Belleville permet également à ces seniors d'être autonomes pour faire leurs courses dans un environnement linguistique familier. Ainsi, il semble difficile de parler d'un isolement des personnes âgées d'origine chinoise lorsque nous tenons compte de la sociabilité au sein des réseaux ethniques. 
En revanche, si les critères d'évaluation de l'isolement reposent sur la fréquence d'interaction entre ces personnes âgées et le reste des membres de la société d'accueil, nous pouvons parler d'une forme d'isolement patent. Faute de maîtriser la langue française et de connaître le système français, ces seniors se retrouvent dépendants de l'aide des autres dans leurs relations avec les institutions françaises. En conséquence, ils témoignent d'une certaine connaissance de leur quartier qu'ils habitent parfois depuis plusieurs décennies et, en même temps, ils ne sont pas en mesure de s'approprier ces lieux et d'être autonomes dans leurs démarches. Ce besoin d'accompagnement administratif laisse penser que les personnes âgées migrantes doivent continuer à justifier de leur existence de manière récurrente, et que la précarité qui les frappe les amène à solliciter les dispositifs sociaux.

\section{La fin de vie et l'au-delà : la question du retour au pays}

Derrière les faits réels que nous pouvons observer dans leurs conditions d'existence - entraves linguistiques, ressenti de discriminations - et qui rendent ces personnes âgées d'origine chinoise invisibles dans l'espace public français, une autre dimension d'ordre symbolique contribue également à leur "invisibilité". Il s'agit de l'image étendue du réseau familial que possèdent ces personnes âgées, qui se déplacent entre leur famille proche résidant en France et leur famille élargie vivant en Chine. En avril 2016, à l'occasion de la fête de Qingming (équivalant de la Toussaint), la moitié des seniors que nous avons rencontrés ont effectué un voyage de retour dans leur pays natal pour commémorer leurs proches décédés et enterrés en Chine. Dans la mesure où ces personnes âgées sont toutes encore autonomes et mobiles, la fréquence de leur retour en Chine varie entre deux fois par an et une fois tous les deux ans. Au pays, tous possèdent encore leur logement d'avant lémigration ${ }^{9}$. L'objectif de leur séjour en Chine est principalement de rendre visite à leurs frères et sœurs encore en vie, de nettoyer les tombes des ancêtres, de voir des praticiens de médecine chinoise ${ }^{10}$ et de préparer leurs funérailles. Parmi ces enquêtés, la plupart ont investi dans une parcelle funéraire au pays natal, "au cas où", en reprenant leurs propres termes.

À la question de savoir si un retour en Chine est envisageable pour leurs très vieux jours, il est répondu négativement dans deux cas sur trois. Parmi le tiers restant, deux tiers répondent qu'ils n’ont encore rien décidé. Cet élément est à mettre au bénéfice des soutiens familiaux que comptent ces personnes âgées, car la majorité des enquêtés envisagent de cohabiter avec leurs enfants en cas de dépendance.

Très distinctement, ceux L'objectif de leur séjour qui se prononcent pour un en Chine est principalement retour au pays projettent de de rendre visite à leurs frères se tourner vers leurs amies et sœurs encore en vie, et leurs connaissances rési- de nettoyer les tombes des dant en Chine.

Par ailleurs, les conditions ancêtres, de voir des praticiens de médecine chinoise et de préparer leurs funérailles. pas toujours un séjour en Chine pour la fin de vie : physiquement, il faudrait que la personne âgée supporte un vol d'une dizaine d'heures entre la France et la Chine ; administrativement, pour certains une demande de visa s'impose pour retourner en Chine; d'autant plus que leurs enfants, étant actifs en France, ne peuvent pas toujours les accompagner pour une fin de vie dans leur pays natal.

Ceux qui sont repartis au pays avant leur décès sont invisibles dans les statistiques démographiques ${ }^{11}$. Quant à ceux qui choisissent de finir leur vie en France, certains manifestent une propension à aller dans des lieux de culte pour "s'apaiser", pour "prier et préparer l'après-vie"12, et à s'investir dans le monde des cultures et des rituels chinois. 


\section{Des lieux de vie aux liens sociaux}

La famille ou les liens familiaux - au sens large semblent être le principal vecteur de la participation de ces personnes âgées à la vie du quartier : aller chercher les petits-enfants à l'école, faire des courses, apprendre le français pour mieux s'occuper de l'ensemble de la famille. Mais lorsque ces personnes âgées deviennent physiquement dépendantes, que faire ? Même si elles restent relativement "invisibles" dans l'espace public, même s'il existe la possibilité qu'elles rentrent au pays avant leur fin de vie, leur prise en charge sanitaire mais aussi sociale avant le retour est une vraie question, comme pour les populations d'autres origines ethniques. Il s'agit de déterminer quelles politiques publiques de la santé et du travail social sont à adapter à la population chinoise du troisième âge, dont les situations varient fortement selon le statut juridique, le sexe, l'origine régionale et le statut social de leurs descendants. Concernant le type de structure adaptée aux personnes âgées immigrées, il existe des foyers-résidences à destination des seniors isolés originaires d'autres populations ethniques. Reste à savoir si ce type d'accueil et de prise en charge peut être envisagé pour les Chinois. Il importe également de repenser les modalités de communication entre les personnes âgées chinoises, les membres de leur famille, les agents des services administratifs et sociaux et les personnels soignants. Enfin, nous souhaitons que ces deux enquêtes suscitent à l'avenir un intérêt pour les personnes âgées d'origine chinoise chez les acteurs associatifs, les chercheurs et les responsables politiques.

10. Sur les recours aux soins alternatifs (automédication, soins en médecine chinoise) des migrants chinois en France, voir Simeng Wang, "Enfant abandonné en Chine, puis domestique en France ?", op. cit. 11. Voir Statistiques d'état civil sur les décès selon le pays de naissance. Voir le site de l'Insee (thème “Décès - Mortalité - Espérance de vie) : www.insee.fr/fr/themes/detail.asp? ref id=ir-irsocsd20133. 12. Par exemple, à l'église protestante chinoise de Paris, à l'église évangélique des Chinois à Paris. Sur la réligion des Chinois de France, voir Hua Hua, "Bali huaren liuxuesheng xinyang jidujiao tezheng yanjiu - yi bali bufen huaren jidujiaohui zhong de liuxuesheng weili” (Études des caractéristiques des étudiants chinois chrétiens à Paris : le cas des étudiants dans certaines églises chrétiennes chinoises à Paris), Qingnian yanjiu [Études de la jeunesse], 6, 2009, pp. 64-73. Zhe Ji, "Territoires migratoires et lieux religieux : cartes des religions des Chinois en Île-de-France”, in Lucine Endelstein, Sébastien Fath, et Séverine Mathieu (dir.), Dieu change en ville. Religion, espace, immigration, Paris, L’Harmattan, 2010, pp. 137-155. 